Anaesthesist 2012 $\cdot 61: 397-398$

DOI 10.1007/s00101-012-2042-0

Online publiziert: 17. Mai 2012

๑) Springer-Verlag 2012

C. Byhahn ${ }^{1} \cdot$ V. Dörges ${ }^{2} \cdot$ B.M. Graf ${ }^{3}$

${ }^{1}$ Klinik für Anästhesiologie, Intensivmedizin und Schmerztherapie, Klinikum der J.W. Goethe-Universität, Frankfurt

${ }^{2}$ Klinik für Anästhesiologie und Operative Intensivmedizin, Universitätsklinikum Schleswig-Holstein Campus Kiel

${ }^{3}$ Klinik für Anästhesiologie, Klinikum der Universität Regensburg

\title{
Maskenbeatmung vor Relaxation
}

\section{Vom Dogma zur Individualität}

In der aktuellen Ausgabe von Der Anaesthesist hinterfragen die beiden Schweizer Kollegen Jacomet und Schnider kritisch eines der grundlegenden Dogmen in der klinischen Anästhesie, nämlich die Forderung, im Rahmen der Narkoseeinleitung vor Gabe eines Muskelrelaxans stets zu überprüfen, ob die Beatmung des Patienten mithilfe der Gesichtsmaske möglich ist.

\section{Dogmen in der Medizin}

Mit einem Dogma verband bereits die antike Philosophie im 1. Jh. n. Chr. einen Begriff für Klarheit und Eindeutigkeit und somit eine nichthinterfragbare Diskussions-, Lebens- oder Handlungsgrundlage. Tiefer gehende oder gar auf Evidenz fußende Begründungen waren und werden bei der Aufstellung eines Dogmas üblicherweise nicht als Entscheidungsgrundlage herangezogen; man handelt quasi ex cathedra. Als Beispiel gilt hierfür in der modernen Anästhesiologie und Intensivmedizin die vermeintlich nephroprotektive Wirkung von Dopamin, die sich auf einer tierexperimentellen Studie an 20 Hunden gründet [1], daraufhin rasch den Weg von der wissenschaftlichen Hypothese über das Paradigma zum langjährigen Dogma angetreten hat und schließlich als Mythos in den Weiten der evidenzbasierten Medizin verglüht ist. Nicht anders verhielt es sich mit dem Krikoiddruck im Rahmen der „ra- pid sequence induction" (RSI): Aus einer Observationsstudie an 26 Patienten entwickelte sich in kurzer Zeit ein nahezu 50 Jahre währendes anästhesiologisches Dogma [2].

Doch die Zeiten ändern sich, und wir ändern uns mit ihnen. Dies galt sowohl für die Verwendung von Dopamin in der Intensivmedizin [3] wie auch für den Krikoiddruck [4] - und jetzt gerät auch noch das Dogma der Maskenbeatmung vor Relaxation bedenklich ins Wanken. O tempora, o mores! Ist denn plötzlich alles falsch, was wir seit der ersten Empfehlung in der anästhesiologischen Literatur, man solle vor der Relaxierung stets die Maskenbeatmung überprüfen [5], glaubten, richtig gemacht zu haben?

\section{$\checkmark$ Waren unsere Erfahrungen mit der Maskenbeatmung vor Relaxierung wirklich sinnlos, gefährlich, ja vielleicht sogar falsch?}

Falsch und richtig sind jedoch Begriffe aus der klassischen, zweiwertigen Logik - ein Sachverhalt kann in diesem Gedankensystem entweder nur richtig oder nur falsch sein. In der Medizin träfe dies beispielsweise auf die Fragestellung "Fraktur" oder „keine Fraktur" zu. Selten führt allerdings dieses Prinzip der reinen Zweiwertigkeit in der modernen Medizin zum Ziel. Vielmehr müssen hier mehrwertige Logiken oder sogar solche mit unendlich vielen Wahrheitswerten („fuzzy lo- gic") angewendet werden. So verhält es sich auch mit der Maskenbeatmung vor Relaxation.

In einer zweiwertigen Logik würde das Dogma, zunächst die Maskenbeatmung zu überprüfen und erst bei einem positiven Ergebnis zu relaxieren, bedeuten, jeden aspirationsgefährdeten Patienten statt mithilfe der RSI zukünftig fiberoptisch wach zu intubieren - schließlich erfolgt bei der RSI die Relaxation ohne vorherige Überprüfung der Maskenbeatmung. Dass dies nicht dem klinischen Vorgehen und dem Verständnis von Anästhesie entspricht, leuchtet ein. Warum überprüfen wir oftmals dennoch die Maskenbeatmung bei elektiven Narkoseeinleitungen und v. a.: mit welcher Konsequenz?

$\checkmark$ Der Glaube, man könne einen prämedizierten Patienten nach Gabe eines potenten Opioids und eines Hypnotikums innerhalb der Hypoxietoleranz wieder in die Spontanatmung überführen, ist ein Irrglaube - und ein sehr gefährlicher noch dazu.

Und seien Sie ehrlich: Wie oft haben Sie allein aufgrund erschwerter oder gar unmöglicher Maskenbeatmung tatsächlich den „Weg zurück“ eingeschlagen und die Narkose wieder ausgeleitet? Noch nie? Das wird sicher die häufigste Antwort sein. 


\section{Der Weg nach vorn ...}

Wie sieht jedoch dann in einem Fall der erschwerten oder unmöglichen Maskenbeatmung der "Weg nach vorn“ in der täglichen Praxis aus? Durch Relaxation lässt sich zumeist die Qualität der Maskenbeatmung deutlich verbessern. In einer Studie an über 12.000 Patienten sah der Algorithmus daher die sofortige Gabe von Succinylcholin bei Problemen mit der Maskenbeatmung vor. War danach die Maskenbeatmung prinzipiell möglich, erfolgte die Intubation des Patienten. Im Fall der persistierenden Unmöglichkeit der Maskenbeatmung wurde zur Sicherstellung der Ventilation zunächst eine Larynxmaske eingelegt. Dies war jedoch gerade einmal bei 2 Patienten erforderlich [6]. In einer weiteren Studie an über 53.000 Patienten lag die Inzidenz der unmöglichen Maskenbeatmung bei 0,15\%. Lediglich 3 dieser 77 Patienten konnten nicht intubiert werden und wurden nach Rückkehr zur Spontanatmung fiberoptisch intubiert oder tracheotomiert. Die Situation des „cannot intubate - cannot ventilate" trat lediglich bei einem Patienten auf und machte eine Koniotomie notwendig. Interessanterweise hatten - trotz unmöglicher Maskenbeatmung - nahe$\mathrm{zu}$ alle Patienten ein Muskelrelaxans erhalten, zumeist Succinylcholin. Von den Autoren wird die frühzeitige Relaxation als ein wesentlicher Schlüssel zum hohen Intubationserfolg angesehen, da zumeist bereits beim ersten Intubationsversuch optimale Bedingungen bestanden [7] Für den Fall der unmöglichen Maskenbeatmung werden also alternative Wege vorgeschlagen, die in den allgemein bekannten "Airway“-Algorithmen festgeschrieben werden sollten, um adäquat geübt werden zu können.

Der „Weg nach vorn“ ist erwiesenermaßen ein erfolgversprechender Lösungsansatz in der Fuzzy logic des Atemwegsmanagements. Warum also überhaupt noch die Maskenbeatmung überprüfen, bevor der Patient relaxiert wird? Auch wenn hierfür - legt man allein die Evidenz zugrunde - die Rationale fehlt, vermittelt dieses Vorgehen insbesondere Berufsanfängern in unserem Fachgebiet den notwendigen Respekt vor dem Einsatz einer Medikamentgruppe, die in- nerhalb weniger Minuten potenziell letale Wirkungen entfalten kann. Auch wenn die unterschwellige Angst am Anfang der anästhesiologischen Tätigkeit vor den Folgen einer Relaxation mit zunehmender Erfahrung schwindet, darf dies keinesfalls dazu führen, dass damit auch der grundsätzliche Respekt vor diesen Substanzen verloren geht.

Maskenbeatmung vor Relaxation - ein anästhesiologisches Dogma verblasst. Es verblasst jedoch nur und verschwindet nicht völlig, und das ist auch gut so. Denn genauso wenig, wie das Dogma der Maskenbeatmung vor Relaxierung evidenzbasiert ist, würde auch ein Dogma der Relaxierung vor Maskenbeatmung jeder Evidenz entbehren. Somit wird aus dem Prinzip der Zweiwertigkeit Fuzzy logic, die uns einerseits eine Richtung vorgibt, aber dennoch genügend Platz für eine patientenadaptierte und individualisiere Entscheidung lässt.

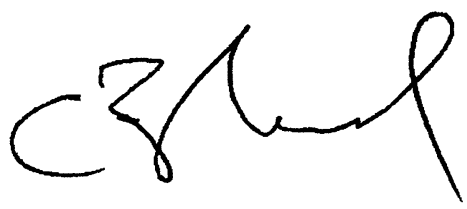

C. Byhahn

\section{Korrespondenzadresse}

Prof. Dr. C. Byhahn

Klinik für Anästhesiologie, Intensivmedizin und Schmerztherapie,

Klinikum der J.W. Goethe-Universität

Theodor-Stern-Kai 7, 60590 Frankfurt

c.byhahn@em.uni-frankfurt.de

Interessenkonflikt. Der korrespondierende Autor gibt für sich und seine Koautoren an, dass kein Interessenkonflikt besteht.

\section{Literatur}

1. McNay JL, McDonald RH, Goldberg RI (1965) Direct renal vasodilation produced by dopamine in the dog. Circ Res 16:510-517

2. Sellick BA (1961) Cricoid pressure to control regurgitation of stomach contents during induction of anaesthesia. Lancet 278:404-406

3. Timmermann A, Byhahn C (2009) Krikoiddruck. Schützender Handgriff oder etablierter Unfug? Anaesthesist 58:663-664

4. Jones D, Bellomo R (2005) Renal-dose dopamine: from hypothesis to paradigm to dogma to myth and, finally, superstition? J Intensive Care Med 4:199-211

5. Stone DJ, Gal TJ (1990) Airway management. In: Miller RD (ed) Anesthesia. Churchill Livingstone, New York, pp 1265-1292

6. Amathieu R, Combes X, Abdi W et al (2011) An algorithm for difficult airway management, modified for modern optical devices (Airtraq laryngoscope; LMA CTrach ${ }^{\mathrm{TM})}$ : a 2-year prospective validation in patients for elective abdominal, gynecologic, and thyroid surgery. Anesthesiology 114:2533

7. Kheterpal S, Martin L, Shanks AM, Tremper KK (2009) Prediction and outcomes of impossible mask ventilation: a review of 50,000 anesthetics. Anesthesiology 110:891-897 